\title{
Musculoskeletal manifestations in polymyalgia rheumatica and temporal arteritis
}

\author{
J Narváez, J M Nolla-Solé, J A Narváez, M T Clavaguera, J Valverde-García, \\ D Roig-Escofet
}

Department of Rheumatology, Hospital Príncipes de España, Ciudad Sanitaria y Universitaria de Bellvitge, Barcelona, Spain

J Narváez

J M Nolla-Solé

M T Clavaguera

J Valverde-García

D Roig-Escofet

Department of CT and MRI, Institut de Diagnòstic per la Imatge, Hospital Duran I Reynals, Ciudad Sanitaria y Universitaria de Bellvitge, Barcelona, Spain

J A Narváez

Rheumatology Unit, Department of Internal Medicine, Delfos Medical Center, Barcelona, Spain

J Narváez

Correspondence to: Dr F J Narváez Garcia, C/ Llobregat no 5, 31 , Hospitalet de Llobregat, 08904 Barcelona, Spain med022409@nacom.es

Accepted 18 April 2001

\begin{abstract}
Objective-To evaluate the incidence and characteristics of musculoskeletal manifestations in polymyalgia rheumatica (PMR) and temporal arteritis (TA). Methods-The records of 163 cases of PMR or TA diagnosed over a 15 year period in one area of Spain were reviewed for the presence and type of musculoskeletal manifestations.

Results-Of 163 patients, 90 had isolated PMR and 73 had TA. Eighteen of the 90 patients $(20 \%)$ with isolated PMR developed distal peripheral arthritis either at diagnosis or during the course of the disease. When it occurred, synovitis was mild, monoarticular or pauci-articular, asymmetrical, transient, and not destructive. Other distal manifestations observed in these patients were carpal tunnel syndrome and distal extremity swelling with pitting oedema. In all cases these manifestations occurred in conjunction with active PMR. As expected, PMR was the most frequent musculoskeletal manifestation in patients with TA, occurring in $56 \%$ of cases. On the contrary, only $11 \%$ of patients with $T A$ developed peripheral arthritis. An important finding was that peripheral arthritis in these patients appears to be linked only temporally to the presence of simultaneous PMR and is not observed in its absence. Distal extremity swelling or defined polyarthritis were not observed.

Conclusion-The spectrum of distal musculoskeletal manifestations of PMR in our series is similar to that reported in other populations. By contrast, distal musculoskeletal symptoms are uncommon in TA. The almost complete absence of distal musculoskeletal manifestations in patients with pure TA suggests different mechanisms of disease in PMR and TA, supporting the view of two separate conditions or one common disease in which host susceptibility influences the clinical expression.

(Ann Rheum Dis 2001;60:1060-1063)
\end{abstract}

There is controversy as to whether polymyalgia rheumatica (PMR) and temporal arteritis (TA) are expressions of the same disease or are two different, partly overlapping, diseases. PMR is a common syndrome of the elderly characterised by pain and stiffness involving the neck, shoulder and pelvic girdles, generally accompanied by constitutional symptoms and a raised erythrocyte sedimentation rate. The cause of musculoskeletal pain in PMR is not completely understood, but inflammation in proximal joints and periarticular structures is a likely basis for much of the discomfort since, to date, there is little evidence to suggest that the musculoskeletal symptoms are related to underlying vasculitis. Evidence of proximal articular and periarticular synovitis has been demonstrated by scanning, MRI, arthroscopy, and synovial biopsy. ${ }^{1-3}$ Moreover, an increasing number of reports have underlined the presence of peripheral synovitis and other distal musculoskeletal manifestations in PMR, suggesting that the spectrum of musculoskeletal involvement in this entity is not completely defined and is broader than has often been thought previously. ${ }^{4-8}$ The clinical predominance of proximal symptoms in PMR has probably overshadowed the less well characterised and more variable distal musculoskeletal manifestations. ${ }^{4}$

TA is a vasculitis of large and medium sized vessels with a predisposition to the cranial arteries in patients older than 50 years. Although cranial and ocular symptoms are the most prominent manifestations in TA, musculoskeletal findings, especially those of PMR, are also common. However, controversy exists over the presence of distal musculoskeletal manifestations in TA and two recent studies have produced conflicting results. Salvarini et al, in a population based study at the Mayo Clinic, reported common and varied distal musculoskeletal symptoms in TA which suggested that the nature of this condition and its clinical expression are broader than has often been considered, and supporting the link between TA and PMR. ${ }^{9}$ By contrast, Gran et al, in a recent prospective study conducted in Norway, noted the particular absence of peripheral arthritis in this group of patients. ${ }^{10}$ The occurrence of peripheral arthritis in PMR and not in TA may reflect different mechanisms of disease.

In view of these contradictory observations, we have reviewed the musculoskeletal manifestations in a well defined cohort of 163 patients with PMR and/or TA diagnosed over a 15 year period in an effort to provide an accurate clinical picture of the frequency and clinical spectrum of these manifestations, and have compared our results with those reported in other major studies of this condition.

\section{Methods}

We retrospectively analysed all patients with PMR and/or TA diagnosed from 1985 to 1999 by the Department of Rheumatology of 
Table 1 foints involved during 20 episodes of peripheral arthritis in 90 patients with isolated polymyalgia rheumatica (PMR) and during eight episodes of peripheral arthritis in 73 patients with temporal arteritis (TA)

\begin{tabular}{lll}
\hline foint & Isolated PMR & TA \\
\hline Knee & $11(55 \%)$ & $4(50 \%)$ \\
Wrist & $7(35 \%)$ & $4(50 \%)$ \\
Metacarpophalangeal & $5(25 \%)$ & $2(25 \%)$ \\
Proximal & & \\
$\quad$ interphalangeal & $3(20 \%)$ & - \\
Elbow & $1(5 \%)$ & $2(25 \%)$ \\
Sternoclavicular & $2(10 \%)$ & $2(25 \%)$ \\
Ankle & - & $1(12.5 \%)$
\end{tabular}

Data are presented as number (\%) of episodes.

Bellvitge Hospital, Barcelona, Spain. The diagnosis of PMR was based on the criteria proposed by Chuang et al. ${ }^{11}$ Patients were considered to have PMR if they met these criteria and had a rapid and persistent response to corticosteroid treatment. The presence of other diseases that might explain the symptoms such as chronic infection, connective tissue diseases, or malignancy excluded the diagnosis of PMR. The diagnosis of TA was made according to the 1990 ACR criteria. ${ }^{12}$ Patients were diagnosed as having TA if they had a positive artery biopsy specimen or, in cases with a negative biopsy or no biopsy, if they fulfilled the remaining four criteria and had a prompt and persistent response to corticosteroid treatment.

After diagnosis all selected patients underwent periodic examinations at the outpatient clinic until death or cessation of treatment and permanent disease remission. All patients were examined by a rheumatologist.

Inpatient and outpatient charts of all patients were reviewed comprehensively to obtain clinical, laboratory, and disease evolution data according to a specifically designed protocol. The end point of patient follow up was the date of the last clinic visit or the date of death. In all selected patients we recorded information on the presence and type of musculoskeletal manifestations, their relationship to the onset and course of the disease, and their response to treatment. We have not included in the study the presence of arthralgias since the high frequency of degenerative disease in this elderly population would make its interpretation difficult. We have also excluded those patients with peripheral arthritis in whom, after examination of joint fluid and/or radiological study, the symptoms could be related to crystal associated arthritis or severe osteoarthritis.

STATISTICAL ANALYSIS

A comparative study between patients with and without musculoskeletal manifestations was performed using the Student's $t$ test for independent continuous variables or the Mann-Whitney U test when the assumption of normality was not realised. To analyse categorical data we performed the $\chi^{2}$ test or the Fisher's exact test when the expected values were less than 5. Statistical significance was defined as $\mathrm{p} \leqslant 0.05$.

Results

From 1985 to 1999 inclusive a total of 163 patients (107 women) were diagnosed with TA and/or PMR. Of these, 73 had TA and 90 had isolated PMR. The mean (SD) age at time of diagnosis for all patients was 72 (8) years (range 51-89) and the mean duration of symptoms prior to the diagnosis was 2.7 (2.6) months. The main clinical features and laboratory data of most of these patients have been extensively reported elsewhere. ${ }^{13}$

\section{PATIENTS WITH ISOLATED PMR}

Eighteen of the 90 patients (20\%) with pure PMR developed clinically detectable peripheral synovitis in whom crystal arthritis and osteoarthritis were excluded. Table 1 lists the joints involved. These patients presented nondeforming monoarthritis or oligoarthritis involving mainly wrists and knees or, less frequently, the sternoclavicular joints, elbows, or some metacarpophalangeal (MCP) or proximal interphalangeal (PIP) joints. None of them developed defined polyarthritis during the study. All of the patients presented with peripheral manifestations at the time of diagnosis and only two of the 18 had a second episode of peripheral symptoms when PMR relapsed. None of these patients presented with peripheral manifestations while proximal symptoms of PMR were absent.

Synovitis was transient and mild, usually asymmetrical, and resolved completely after corticosteroid therapy was started or the prednisone dose was increased. Rheumatoid factor was negative in all cases. No erosive changes or juxta-articular osteoporosis were seen on plain radiographs of affected joints. Two patients (2\%) with synovitis of the wrists referred symptoms of acute carpal tunnel syndrome. Finally, only one of the 18 patients $(1 \%)$ developed distal symmetrical swelling of the upper limbs with pitting oedema over the dorsum of the hands and wrists concurrently with proximal PMR symptoms. In this patient MRI examination showed severe extensor tenosynovitis with peritendinous oedema, without evidence of concomitant wrist or hand joint synovitis. HLA B7 was negative. Corticosteroids were given and the swelling responded promptly. No residual contractures were observed.

\section{PATIENTS WITH TEMPORAL ARTERITIS}

Forty one of the 73 patients (56\%) developed PMR at some time during the course of their TA. As in patients with pure PMR, shoulder pain was the most frequent feature being observed in $100 \%$ of the patients, while the hips and neck were less commonly affected.

In 33 patients PMR began concurrently $(<1$ month from the diagnosis of TA), in one PMR began at some time after TA was diagnosed as a result of one relapse, and in seven patients PMR began before TA. These seven patients were originally diagnosed as having isolated PMR because none presented with clinical evidence of TA (in none of them was a temporal artery biopsy specimen taken at the time of diagnosis). One suffered an arteritic recurrence 26 months after the end of a 29 month course of treatment. The remaining six experienced an arteritic relapse during the course of steroid 
Table 2 Comparison between patients with and without distal musculoskeletal manifestations

\begin{tabular}{|c|c|c|c|}
\hline (A) Patients with isolated PMR & $\begin{array}{l}\text { Patients with distal } \\
\text { musculoskeletal manifestations } \\
n=18(20 \%)\end{array}$ & $\begin{array}{l}\text { Patients without distal } \\
\text { musculoskeletal manifestations } \\
n=72(80 \%)\end{array}$ & p Value \\
\hline Mean (SD) age at onset of disease (years) & $71.3(9)$ & $71.8(8)$ & NS \\
\hline $\mathrm{F}: \mathrm{M}$ ratio & $12 / 6(2)$ & $46 / 26(1.8)$ & NS \\
\hline Malaise/anorexia/weight loss & $9(50 \%)$ & $40(55 \%)$ & NS \\
\hline Low grade fever & $1(6 \%)$ & $6(8 \%)$ & NS \\
\hline Mean (SD) ESR (mm/h) & $72(23)$ & $74(20)$ & NS \\
\hline Mean (SD) haemoglobin $(\mathrm{g} / \mathrm{l})$ & $116(13)$ & $118(12)$ & NS \\
\hline Raised alkaline phosphatase & $4(22 \%)$ & $12(17 \%)$ & NS \\
\hline Raised ALT/AST & $2(11 \%)$ & $6(8 \%)$ & NS \\
\hline (B) Patients with TA & $\begin{array}{l}\text { Patients with musculoskeletal } \\
\text { manifestations } n=41(56 \%)\end{array}$ & $\begin{array}{l}\text { Patients without } \\
\text { musculoskeletal manifestations } \\
n=32(44 \%)\end{array}$ & p Value \\
\hline Mean (SD) age at onset of disease (years) & $71.4(8)$ & $73(9)$ & NS \\
\hline $\mathrm{F}: \mathrm{M}$ ratio & $28 / 13(2.1)$ & $21 / 11(1.9)$ & NS \\
\hline Headache & $41(100 \%)$ & $32(100 \%)$ & NS \\
\hline Abnormal temporal artery & $35(85 \%)$ & $29(90 \%)$ & NS \\
\hline Jaw claudication & $17(41 \%)$ & $8(25 \%)$ & NS \\
\hline Malaise/anorexia/weight loss & $27(66 \%)$ & $19(59 \%)$ & NS \\
\hline Fever & $7(17 \%)$ & $2(6 \%)$ & NS \\
\hline Amaurosis fugax & $10(24 \%)$ & $4(12 \%)$ & NS \\
\hline Mean $(\mathrm{SD}) \operatorname{ESR}(\mathrm{mm} / \mathrm{h})$ & $91(24)$ & $80(21)$ & NS \\
\hline Mean (SD) haemoglobin (g/l) & $110(13)$ & $115(14)$ & NS \\
\hline Raised alkaline phosphatase & $10(24 \%)$ & $7(22 \%)$ & NS \\
\hline Raised ALT/AST & $5(12 \%)$ & $3(9 \%)$ & NS \\
\hline
\end{tabular}

$\mathrm{PMR}=$ polymyalgia rheumatica; $\mathrm{TA}=$ temporal arteritis; $\mathrm{ESR}=$ erythrocyte sedimentation rate; ALT/AST = alanine aminotransferase/aspartate aminotransferase; $\mathrm{SD}=$ standard deviation.

treatment (median time to relapse from initiation of therapy 26 (16) weeks). Relapses usually occurred during the tapering of the prednisone dose or as a consequence of an unauthorised discontinuation of treatment $(n=1)$. In these seven cases arteritis was confirmed by a positive biopsy specimen.

Eight of the 73 patients with TA $(11 \%)$ developed clinically detectable synovitis. Interestingly, all of these patients also had PMR. Table 1 lists the joints involved. In six a non-deforming, self-limited mono or asymmetrical oligoarthritis was observed, affecting mainly knees and wrists or, less frequently, elbows or some MCP or PIP joints. The remaining two patients presented with tenderness and mild swelling of both sternoclavicular joints. No erosive changes were seen on plain radiographs of the affected joints, although erosions in the sternoclavicular joints were confirmed by tomography in two patients. All of the eight patients developed peripheral manifestations concurrently with the time proximal PMR began. In all cases synovitis was transient and this manifestation resolved completely after corticosteroid treatment was started. Clinical symptoms suggesting carpal tunnel syndrome were observed in one patient (1\%) with synovitis of the wrists. Distal extremity swelling, tenosynovitis, or defined polyarthritis were not observed in any patient.

COMPARISONS BETWEEN PATIENTS WITH AND WITHOUT MUSCULOSKELETAL MANIFESTATIONS At the time of diagnosis patients with PMR and distal musculoskeletal manifestations did not differ from those without these features. In comparing patients with TA and musculoskeletal symptoms with those without, we observed that the group with musculoskeletal findings seemed to have more severe disease characterised by a greater increase in erythrocyte sedimentation rate, lower values of haemoglobin, and an increased incidence of constitutional symptoms, jaw claudication, and visual problems, although these differences were no longer statistically significant (table 2).

\section{Discussion}

There is considerable controversy regarding the frequency of peripheral synovitis in PMR since its reported incidence varies considerably, ranging from $6 \%$ to $60 \%$ in various series. ${ }^{4-7}$ These discrepancies may be attributed to the variable use of different diagnostic criteria for PMR (with important selection bias in the ascertainment of PMR cases), the definition of synovitis (which has been equated with arthralgia in some studies), and difficulty in the interpretation of scans and radiographs due to coexisting degenerative disease. In our series, $20 \%$ of patients with isolated PMR developed clinically detectable peripheral arthritis either at diagnosis or during the course of the disease. This percentage is very similar to those reported in previous observations. ${ }^{457}$ In all of these cases peripheral synovitis occurred in conjunction with active PMR, particularly at its onset or, less frequently, during a relapse. The data suggest that synovitis is not uncommon in PMR, and seems to be a main contributing factor to many of the symptoms seen in patients with this condition. When it occurred, synovitis presented as mono or asymmetrical oligoarthritis involving mainly wrists and knees. Unlike rheumatoid arthritis, synovitis was transient and mild, non-deforming, and resolved completely after corticosteroid treatment was started or the prednisone dose was increased. None of the patients developed defined polyarthritis. Rheumatoid factor was negative in all cases. No erosive changes or juxta-articular osteoporosis were seen on plain radiographs of affected joints. Although it has been suggested that the presence of peripheral 
arthritis in PMR is associated with a longer duration of steroid treatment and a higher frequency of relapses indicating a subset with more severe disease,${ }^{5}$ in our series patients with PMR and distal musculoskeletal manifestations did not differ from those without them.

Other distal manifestations observed in patients with isolated PMR were carpal tunnel syndrome and distal extremity swelling with pitting oedema. The incidence of carpal tunnel syndrome described in previous reports ranges from $6 \%$ to $14 \%$, being mainly attributed to wrist flexor tenosynovitis. ${ }^{4-611}$ In our population this percentage was substantially lower $(2 \%)$ and in these patients carpal tunnel syndrome resulted mainly from synovitis of the wrists. Distal extremity swelling with pitting oedema represents another clinical feature recently recognised in PMR; in our series only $1 \%$ of patients with isolated PMR had distal swelling with pitting oedema compared with an incidence of $8-12 \%$ in earlier reports. ${ }^{458} \mathrm{We}$ cannot completely explain the low incidence of distal extremity swelling in our patients. A retrospective review such as this is inevitably associated with a bias toward underreporting of these features. In this regard, it is reasonable to assume that some manifestations, especially those less recognisable or unfamiliar, may have been overlooked. However, the patients were carefully evaluated at regular intervals and, since the presence of any of these manifestations generally implies significant pain and functional impairment of the patient and are easily detectable on physical examination, it seems likely most occurrences would have been reported in the medical records and therefore included in this study. Alternatively, this difference may simply reflect the variability in musculoskeletal manifestations of PMR in different populations. In this sense, our findings are in close agreement with Gran et al who also observed a low frequency of distal pitting oedema $(0.4 \%)$ in a recent prospective study conducted in Norway. ${ }^{10}$ The reason for this variability among different populations may well be genetic.

As expected, PMR with proximal aching and morning stiffness was the most frequent musculoskeletal manifestation in patients with TA, occurring in $56 \%$ of cases. In addition to the frequent proximal symptoms, only $11 \%$ of patients with TA developed distal peripheral arthritis. This percentage was lower than that reported in a population based study recently conducted at the Mayo Clinic (23\%). ${ }^{9}$ The clinical characteristics and distribution of peripheral joint inflammation observed in these patients were similar to those described in the group with isolated PMR. With the exception of the sternoclavicular joints, no erosive changes were seen. In comparing patients with musculoskeletal symptoms with those without, we observed that the group with peripheral arthritis seemed to have more severe disease characterised by more laboratory abnormalities reflecting inflammation and increased incidence of constitutional symptoms, jaw claudication, and visual problems. Interestingly, an important finding was that peripheral joint involvement was only present in conjunction with active PMR and was not observed when PMR was absent. The particular absence of peripheral arthritis in patients with isolated TA has also been observed previously by several authors ${ }^{10}$ and may, to some extent, indicate important differences in the aetiopathogenesis of PMR and TA. Moreover, distal extremity swelling with pitting oedema was not observed in any case and, contrary to other reports that have described both seronegative and seropositive rheumatoid arthritis in patients with TA, ${ }^{91415}$ none of our patients developed defined polyarthritis during the study.

In summary, although the frequency of distal musculoskeletal manifestations observed in our patients with PMR was different from that given in some previous reports, overall the findings of this study support and confirm the similar findings found in different populations with this condition. However, in contradiction to another major study of this condition, ${ }^{9}$ we found that distal musculoskeletal manifestations in patients with TA were uncommon. Peripheral synovitis in these patients appears to be linked only temporally to the presence of simultaneous PMR. The almost complete absence of peripheral arthritis and other distal musculoskeletal manifestations in patients with pure TA suggests different mechanisms of disease in PMR and TA, supporting the view of two separate conditions or one common disease in which host susceptibility influences the clinical expression. Further studies in other populations are needed to confirm our results.

1 O'Duffy JD, Wahner HW, Hunder GG. Joint imaging in polymyalgia rheumatica. Mayo Clin Proc 1976;51:519-24. 2 pouglas WA, Martin BA, Morris JH. Polymyalgia rheumatica: an arthroscopic study of the shoulder joint. rheumatica: an arthroscopic study
Ann Rheum Dis 1983;42:311-6.

3 Ann Rheum Dis 1983;42:311-6. active polymyalgia rheumatica. Ann Intern Med 1997;127: $27-31$.

4 Salvarini C, Cantini F, Olivieri I, Hunder GG. Polymyalgia heumatica: a disorder of extraarticular synovial structures?. J Rheumatol 1999;26:517-21.

5 Salvarini C, Cantini F, Macchioni P, et al. Distal musculoskeletal manifestations in polymyalgia rheumatica. A prosp

6 Healey LA. Long-Term follow-up of polymyalgia rheumatica: evidence for synovitis. Semin Arthritis Rheum 1984;13:322-8.

7 Al-Hussaini AS, Swannell AJ. Peripheral joint involvement in polymyalgia rheumatica: a clinical study of 56 cases. Br J Rheumatol 1985;24:27-30.

8 Salvarini C, Gabriel S, Hunder GG. Distal extremity swelling with pitting edema in polymyalgia rheumatica. Report of nineteen cases. Arthritis Rheum 1996;39:73-80.

9 Salvarini C, Hunder GG. Musculoskeletal manifestations in a population-based cohort of patients with giant cell arteritis. Arthritis Rheum 1999;42:1259-66.

10 Gran JT, Myklebust G. The incidence and clinical characteristics of peripheral arthritis in polymyalgia rheumatica and temporal arteritis: a prospective study of 231 cases. Rheumatology 2000;39:283-7.

11 Chuang TY, Hunder GG, Ilstrup DM, Kurland LT. Polymyalgia rheumatica. A 10 year epidemiologic and clinical study. Ann Intern Med 1982;97:672-80.

12 Hunder GG, Bloch DA, Michel BA, et al. The American College of Rheumatology 1990 criteria for the classification College of Rheumatology 1990 criteria for the classification
of giant cell arteritis. Arthritis Rheum 1990;33:1122-8.

13 Narváez J, Nolla-Solé JM, Clavaguera MT, Valverde-Garcia J, Roig-Escofet D. Long-term therapy in polymalgia heumatica: effect of coexistent temporal arteritis. J Rheumatol 1999;26:1949-52.

14 Omdal R, Husby G. Coexistence of temporal arteritis/ polymyalgia rheumatica and rheumatoid arthritis. Clin Rheumatol 1984;3:525-7.

15 Hall S, Ginsburg WW, Vollertsen RS, Hunder GG. The coexistence of rheumatoid arthritis and giant cell arteritis. J Rheumatol 1983;10:995-7. 